\title{
Wavelet multiresolution complex network for analyzing multivariate nonlinear time series
}

\author{
Zhong-Ke Gao ${ }^{*}$ \\ School of Electrical and Information Engineering, Tianjin University, \\ Tianjin 300072, China \\ zhongkegao@tju.edu.cn \\ Shan Li, Wei-Dong Dang, Yu-Xuan Yang \\ School of Electrical and Information Engineering, Tianjin University, \\ Tianjin 300072, China \\ Younghae Do \\ Department of Mathematics, KNU-Center for Nonlinear Dynamics, Kyungpook National University, \\ Daegu, 41566, Republic of Korea \\ Celso Grebogi \\ Institute for Complex Systems and Mathematical Biology, Kings College, University of Aberdeen, \\ Aberdeen AB24 3UE, UK
}

\begin{abstract}
Characterizing complicated behavior from time series constitutes a fundamental problem of continuing interest and it has attracted a great deal of attention from a wide variety of fields on account of its significant importance. We in this paper propose a novel wavelet multiresolution complex network (WMCN) for analyzing multivariate nonlinear time series. In particular, we first employ wavelet multiresolution decomposition to obtain the wavelet coefficients series at different resolutions for each time series. We then infer the complex network by regarding each time series as a node and determining the connections in terms of the distance among the feature vectors extracted from wavelet coefficients series. We apply our method to analyze the multivariate nonlinear time series from our oil-water two-phase flow experiment. We construct various wavelet multiresolution complex networks and use the weighted average clustering coefficient and the weighted average shortest path length to characterize the nonlinear dynamical behavior underlying the derived networks. In addition, we calculate the permutation entropy to support the findings from our network analysis. Our results suggest that our method allows characterizing the nonlinear flow behavior underlying the transitions of oil-water flows.
\end{abstract}

Keywords: Nonlinear time series analysis; Wavelet multiresolution; Complex network; Oil-water flows

\section{Introduction}

Characterizing dynamical processes in a time-dependent complex system from observed time series of just one or at most a few variables is a fundamental problem of significant importance in many research fields.

*Author for correspondence 
Different nonlinear time series analysis methods have been developed to fulfill this challenging task, e.g., chaotic analysis [Daw et al., 1995], permutation entropy [Bandt \& Pompe, 2002], recurrence plot [Marwan et al., 2007], and time-frequency representation [Gao et al., 2016]. Nonlinear time series analysis has been broadly adopted in scientific research and engineering applications. Many theoretical developments and new methods for nonlinear time series analysis have significantly contributed to the understanding of complex systems. Complex network originates from graph theory, which is an abstract description of the complex system, consisting of nodes and the edges between the nodes [Wang et al., 2016; Boccaletti et al., 2014; Zou et al., 2015; Kim et al., 2013; Huang et al., 2014; Lu et al., 2013; Kenett et al., 2015; Lu \& Chen, 2005; Feldhoff et al., 2012]. Recently, the complex network theory has been incorporated into the analysis of time series and many successful applications have been achieved in different research fields [Wang et al., 2016; Zhang et al., 2006; 2008; Xu et al., 2008; Lacasa et al., 2008; Marwan et al., 2009; Majhi et al., 2016; Wang et al., 2015; Xiang et al., 2012; Marwan \& Kurths, 2015; Peng et al., 2013; Wang et al., 2012; Huang et al., 2012; Livi et al., 2016; Wang et al., 2011; Donges et al., 2012; Gao et al., 2015; 2016; 2017; Shen et al., 2014; Jachan et al., 2009; Nakamura et al., 2016], including turbulence [Liu et al., 2010; Scarsoglio et al., 2016], brain network [Zhang et al., 2016], traffic network [Tang et al., 2016], climate network [Donges et al., 2011], sunspots [Zou et al., 2014] and multiphase flows [Gao et al., 2013; Mosdorf \& Górski, 2015], etc. Despite the existing results [Schelter et al., 2014], network-based methods dedicated to analyzing multivariate nonlinear time series still remain much to be developed. Moreover, most of the complex network inference methods do not pay much attention to the characteristics of the time series at different resolutions, which is of great value in the analysis of multivariate nonlinear time series. And in multiresolution analysis domain, the wavelet transform is a powerful mathematical tool which can not only get the frequency domain characteristics (corresponding to the global characteristics) of the signal, but also the local characteristics. In addition, one of the advantages of wavelet transform techniques is the ability to deal with non-stationary time series, which are very common in the analysis of multivariate nonlinear time series, given the continuous presence of abrupt changes and volatility. The wavelet analysis has been widely incorporated into the analysis of time series and many successful applications have been achieved in different research fields [Kao et al., 2013; Jammazi., 2012; He et al., 2012; Pal \& Mitra, 2009; Goyal \& Mehra, 2017]. In this regard, we in this paper propose a novel wavelet multiresolution complex network (WMCN), which enables to characterize dynamical behavior from multivariate nonlinear time series at different resolutions. We take the multivariate nonlinear time series from experimental oil-water flows as an example to demonstrate the effectiveness of our approach. Vertical oil-water two-phase flow exists in a wide range of industrial applications, such as petroleum industry, chemical industry and energy. The multiphase flow [Gao et al., 2013; 2016; Deising et al., 2015] is a typical nonlinear dynamic system and numerous studies have been carried out. In particular, characterization of nonlinear flow behavior from flow signals has attracted a lot of attention. Moreover, as the appearance of multi-electrode sensor [Gao et al., 2016], the acquisition of multi-channel flow signals becomes possible. However, the sensor measurements acquired from oil-water flows usually consist of lots of non-stationary multi-resolution components, which bring many difficulties for the characterization of complex dynamical behaviors in such a complex system. In this regard, a novel method is required to realize the multi-channel information fusion and multivariate nonlinear time series analysis. We demonstrate that our WMCN method enables to fulfill this challenging task. We infer and analyze WMCN from experimental multivariate flow signals at different resolutions. The results suggest that our method is capable of obtaining the essential and important flow information at different resolutions and further allows uncovering the complicated nonlinear flow dynamical behaviors governing the transitions of three typical oil-in-water flow patterns. We also carry out the permutation entropy analysis to demonstrate the effectiveness of WMCN. The combination of wavelet multiresolution technique and multivariate complex network opens up new venues to address the challenge in multivariate nonlinear time series.

\section{Methodology}

Wavelet multiresolution technique allows us to decompose a time series into some wavelet coefficients series, including details and approximations. As an efficient signal processing method, wavelet multiresolution 
technique has been widely used in image processing [Tee et al., 2011], EEG signal analysis [Guler \& Ubeyli, 2005], etc. For a multivariate nonlinear time series $\left\{x_{k, l}\right\}_{l=1}^{L}, k=1,2, \ldots, S$ with $S$ sub-signal of equal length $L$, we first use the wavelet multiresolution technique to obtain the wavelet coefficients series at different resolutions for each time series. All wavelet transforms can be specified in terms of a low-pass filter $h$, which satisfies the standard quadrature mirror filter condition:

$$
H(z) H\left(z^{-1}\right)+H(-z) H\left(-z^{-1}\right)=1,
$$

where $H(z)$ denotes the $z$-transform of the filter $h$. Correspondingly, the high-pass filter $g$ can be defined as:

$$
G(z)=z H\left(-z^{-1}\right) .
$$

A sequence of filters with increasing length (indexed by $i$ ) can be obtained:

$$
\begin{aligned}
& H_{i+1}(z)=H\left(z^{2 i}\right) H_{i}(z), \quad i=0, \ldots, I-1, \\
& G_{i+1}(z)=G\left(z^{2 i}\right) H_{i}(z), \quad i=0, \ldots, I-1 .
\end{aligned}
$$

The normalized wavelet and resolution basis function can be defined as

$$
\begin{aligned}
& \Phi_{i, l}(k)=2^{i / 2} h_{i}\left(k-2^{i} l\right), \\
& \Psi_{i, l}(k)=2^{i / 2} g_{i}\left(k-2^{i} l\right),
\end{aligned}
$$

where the factor $2^{i / 2}$ is an inner product normalization, $i$ and $l$ are the resolution parameter and the translation parameter, respectively. Therefore, the discrete wavelet transform decomposition can be described as:

$$
\begin{aligned}
& C A^{i}(l)=x(k) \Phi_{i, l}(k), \\
& C D^{i}(l)=x(k) \Psi_{i, l}(k) .
\end{aligned}
$$

In this paper, we choose the Daubechies2 wavelet [Lina \& Mayrand, 1995] and set the maximum resolution as six. Thus a multivariate nonlinear time series $\left\{x_{k, l}\right\}_{l=1}^{L}, k=1,2, \ldots, S$ can be decomposed into six details $\left\{C D_{n}^{i}\right\}^{k}, i=1,2, \ldots, 6, k=1,2, \ldots, S, n=1,2, \ldots, N$ and approximations $\left\{C A_{n}^{i}\right\}^{k}, i=1,2, \ldots, 6$, $k=1,2, \ldots, S, n=1,2, \ldots, N$, where $i$ represents the resolution, and the larger $i$ is, the higher the resolution will be; $N$ is the length of the wavelet coefficients series corresponding to the resolution $i$. We decompose each single time series, for example $\left\{x_{k, l}\right\}_{l=1}^{L}, k=1,2, \ldots, S$, into 12 wavelet coefficients series at six different resolutions and 6 for details, 6 for approximations. Under each resolution, we extract 12 statistical features of wavelet coefficients series as follows:

(a) Maximum of the wavelet coefficients series in each subband:

$$
\begin{aligned}
& x_{k, A 1}^{i}=\max \left\{C A_{n}^{i}\right\}^{k}, \quad i=1,2, \ldots, 6, n=1,2, \ldots, N, k=1,2, \ldots, S, \\
& x_{k, D 1}^{i}=\max \left\{C D_{n}^{i}\right\}^{k}, \quad i=1,2, \ldots, 6, n=1,2, \ldots, N, k=1,2, \ldots, S .
\end{aligned}
$$

(b) Minimum of the wavelet coefficients series in each subband:

$$
\begin{aligned}
& x_{k, A 2}^{i}=\min \left\{C A_{n}^{i}\right\}^{k}, \quad i=1,2, \ldots, 6, n=1,2, \ldots, N, k=1,2, \ldots, S, \\
& x_{k, D 2}^{i}=\min \left\{C D_{n}^{i}\right\}^{k}, \quad i=1,2, \ldots, 6, n=1,2, \ldots, N, k=1,2, \ldots, S .
\end{aligned}
$$


(c) Mean of the wavelet coefficients series in each subband:

$$
\begin{aligned}
& x_{k, A 3}^{i}=\frac{1}{N} \sum_{n=1}^{N}\left\{C A_{n}^{i}\right\}^{k}, \quad i=1,2, \ldots, 6, n=1,2, \ldots, N, k=1,2, \ldots, S, \\
& x_{k, D 3}^{i}=\frac{1}{N} \sum_{n=1}^{N}\left\{C D_{n}^{i}\right\}^{k}, \quad i=1,2, \ldots, 6, n=1,2, \ldots, N, k=1,2, \ldots, S .
\end{aligned}
$$

(d) Standard deviation of the wavelet coefficients series in each subband:

$$
\begin{aligned}
& x_{k, A 4}^{i}=\sqrt{\frac{1}{N-1} \sum_{n=1}^{N}\left(C A_{n}^{i, k}-x_{k, A 3}^{i}\right)^{2}}, \quad i=1,2, \ldots, 6, n=1,2, \ldots, N, k=1,2, \ldots, S, \\
& x_{k, D 4}^{i}=\sqrt{\frac{1}{N-1} \sum_{n=1}^{N}\left(C D_{n}^{i, k}-x_{k, D 3}^{i}\right)^{2}}, \quad i=1,2, \ldots, 6, n=1,2, \ldots, N, k=1,2, \ldots, S .
\end{aligned}
$$

(e) Steepness function of the wavelet coefficients series in each subband:

$$
\begin{aligned}
& x_{k, A 5}^{i}=\frac{1}{(N-1)} \frac{\sum_{n=1}^{N}\left(C A_{n}^{i, k}-x_{k, A 3}^{i}\right)^{3}}{\left(x_{k, A 4}^{i}\right)^{3}}, \quad i=1,2, \ldots, 6, n=1,2, \ldots, N, k=1,2, \ldots, S, \\
& x_{k, D 5}^{i}=\frac{1}{(N-1)} \frac{\sum_{n=1}^{N}\left(C D_{n}^{i, k}-x_{k, D 3}^{i}\right)^{3}}{\left(x_{k, D 4}^{i}\right)^{3}}, \quad i=1,2, \ldots, 6, n=1,2, \ldots, N, k=1,2, \ldots, S .
\end{aligned}
$$

(f) Kurtosis function of the wavelet coefficients series in each subband:

$$
\begin{aligned}
& x_{k, A 6}^{i}=\frac{1}{(N-1)} \frac{\sum_{n=1}^{N}\left(C A_{n}^{i, k}-x_{k, A 3}^{i}\right)^{4}}{\left(x_{k, A 4}^{i}\right)^{4}}, \quad i=1,2, \ldots, 6, n=1,2, \ldots, N, k=1,2, \ldots, S, \\
& x_{k, D 6}^{i}=\frac{1}{(N-1)} \frac{\sum_{n=1}^{N}\left(C D_{n}^{i, k}-x_{k, D 3}^{i}\right)^{4}}{\left(x_{k, D 4}^{i}\right)^{4}}, \quad i=1,2, \ldots, 6, n=1,2, \ldots, N, k=1,2, \ldots, S .
\end{aligned}
$$

Then we construct a new feature vector $\left\{X_{k}^{i}\right\}, i=1,2, \ldots, 6, k=1,2, \ldots, S$ for each single time series as follows

$$
X_{k}^{i}=\left\{\overline{x_{k, A 1}^{i}}, \overline{x_{k, A 2}^{i}}, \overline{x_{k, A 3}^{i}}, \overline{x_{k, A 4}^{i}}, \overline{x_{k, A 5}^{i}}, \overline{x_{k, A 6}^{i}}, \overline{x_{k, D 1}^{i}}, \overline{x_{k, D 2}^{i}}, \overline{x_{k, D 3}^{i}}, \overline{x_{k, D 4}^{i}}, \overline{x_{k, D 5}^{i}}, \overline{x_{k, D 6}^{i}}\right\},
$$

where $\overline{x_{k, A 1}^{i}}$ is the normalized value of $x_{k, A 1}^{i}$ given by the following formula and so do the others:

$$
\overline{x_{k, A 1}^{i}}=\left[x_{k, A 1}^{i}-\min \left(x_{k, A 1}^{i}\right)\right] /\left[\max \left(x_{k, A 1}^{i}\right)-\min \left(x_{k, A 1}^{i}\right)\right], \quad k=1,2, \ldots, S .
$$

We regard each time series as a node and then determine the connections between node $p$ and $q$ in terms of the distance between the feature vectors extracted from time series $p$ and $q$ as follows

$$
\begin{array}{r}
r_{p, q}^{i}=\left\|X_{p}^{i}-X_{q}^{i}\right\|=\sqrt{\sum_{c=1}^{6}\left(\overline{x_{p, A c}^{i}}-\overline{x_{q, A c}^{i}}\right)^{2}+\sum_{c=1}^{6}\left(\overline{x_{p, D c}^{i}}-\overline{x_{q, D c}^{i}}\right)^{2}}, \\
c=1,2, \ldots, 6, p=1,2, \ldots, S, q=1,2, \ldots, S, i=1,2, \ldots, 6 .
\end{array}
$$


The above operations allow us to obtain a distance matrix $R^{i}, i=1,2, \ldots, 6$ for six resolutions. Then we choose the threshold

$$
r r^{i}=M^{i}+n \sigma^{i},
$$

where $M^{i}$ is the mean of the distance matrix $R^{i}, i=1,2, \ldots, 6$ and $\sigma^{i}$ is the corresponding standard deviation and $n=0.10$. We can then obtain the network adjacency matrix $R A_{p q}^{i}$ of the size $S \times S$ at different resolutions $i=1,2, \ldots, 6$ through the following rule:

$$
R A_{p q}^{i}=\left\{\begin{array}{cc}
r_{p q}^{i}, & r_{p q}^{i} \leq r r^{i} \\
0, & r_{p q}^{i}>r r^{i}
\end{array}, \quad p=1,2, \ldots, S, q=1,2, \ldots, S, i=1,2, \ldots, 6\right.
$$

where $R A_{p q}^{i}$ represents the weight of edges in complex networks. That is, an weighted edge between node $p$ and $q$ exists if $r_{p q}^{i} \leq r r^{i}$; otherwise, node $p$ and $q$ are disconnected if $r_{p q}^{i}>r r^{i}$. The topological structure of the derived complex network can be described by the $R A_{p q}^{i}$. Therefore, we can obtain a wavelet multiresolution complex network (WMCN), which consists of six adjacency matrices at six different resolutions.

Our method allows mapping a multivariate nonlinear time series into weighted complex networks at different resolutions, which can be quantitatively characterized via network measures [Antoniou \& Tsompa, 2008]. We use the weighted average clustering coefficient and weighted average shortest path length to characterize the derived weighted complex networks. The weighted average clustering coefficient $C_{R A^{i}}^{i}$ is defined as:

$$
\begin{gathered}
C_{R A^{i}}^{i}=\frac{1}{S} \sum_{v=1}^{S} C_{R A^{i}}(v), \\
C_{R A^{i}}^{i}(v)=\frac{\sum_{p, q} R A_{v p}^{i} R A_{p q}^{i} R A_{q v}^{i}}{\sum_{p, q} R A_{v p}^{i} R A_{q v}^{i}}, \quad p \neq q,
\end{gathered}
$$

where $R A_{v p}^{i}$ is the weight between the node $v$ and $p$, i.e., the element of weighted matrix $R A^{i}, C_{R A^{i}}^{i}(v)$ is the weighted clustering coefficient of each node $v$. The weighted average shortest path length $L_{R A^{i}}^{i}$ is defined as:

$$
\begin{gathered}
L_{R A^{i}}^{i}=\frac{1}{S(S-1)} \sum_{p, q \in S, p \neq q} d_{p q}^{i}, \\
d_{p q}^{i}=\min _{\gamma(p, q) \in \Gamma(p, q)}\left[\sum_{m, n \in \gamma(p, q)} R A_{m n}^{i}\right],
\end{gathered}
$$

where $R A_{p q}^{i}$ is the weight between node $p$ and $q, d_{p q}^{i}$ is the weighted shortest path between node $p$ and $q$, and $\gamma(p, q)$ is a path from node $p$ and $q$ and $\Gamma(p, q)$ is the class of paths from $p$ to $q$.

\section{WMCN analysis of multivariate nonlinear flow signals}

We carry out the vertical oil-water two-phase flow experiment in the multiphase flow facility, where the inner diameter of the Plexiglas pipe is $20 \mathrm{~mm}$. The experimental process is as the follows: first, we fix the water-cut $\left(K_{w}\right)$, and gradually increase the mixture flow velocity $\left(V_{m}\right)$. And then, changing the watercut, we repeat the above operation to generate all flow conditions. The sampling rate is $4 \mathrm{kHz}$ and the sampling duration for each flow condition is $30 \mathrm{~s}$. For each flow condition, a novel HCMC sensor (Highspeed Cycle Motivation Conductance sensor) is employed to acquire 48 channel time series from different spatial locations. The HCMC sensor allows capturing the local oil-in-water flow information in different spaces. Meanwhile, we use the snapshots from high-speed video camera to define different experimental flow patterns. During the experiments, we have observed three oil-in-water flow patterns: slug flow (D $\mathrm{OS} / \mathrm{W})$, bubble flow $(\mathrm{D} \mathrm{O} / \mathrm{W})$ and very fine dispersed flow (VFD O/W). 


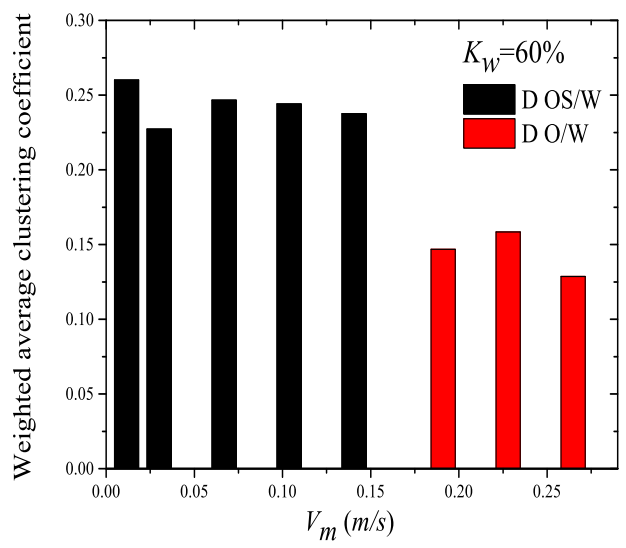

(a)

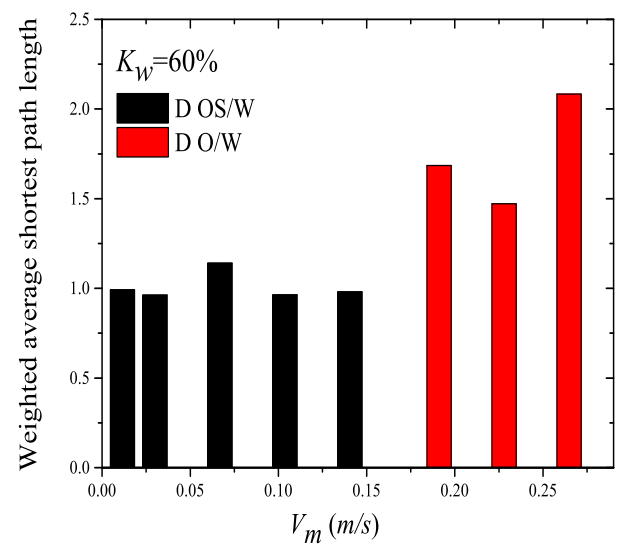

(b)

Fig. 1. Distributions of the network measures with the change of total mixture flow velocity for different flow conditions for a fixed $K_{w}=60 \%$. (a) Weighted average clustering coefficient; (b) Weighted average shortest path length.

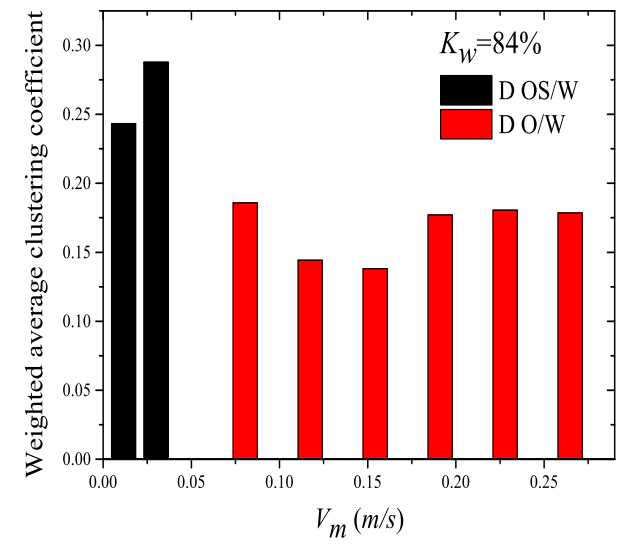

(a)

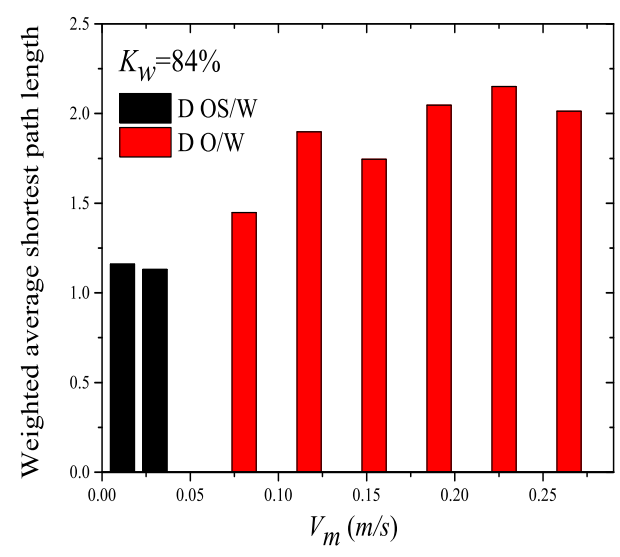

(b)

Fig. 2. Distributions of the network measures with the change of total mixture flow velocity for different flow conditions for a fixed $K_{w}=84 \%$. (a) Weighted average clustering coefficient; (b) Weighted average shortest path length.

We implement our WMCN method to analyze the multivariate nonlinear flow signals. In particular, we infer various complex networks at different resolutions for each flow condition. Note that, we choose the proper resolution for different flow patterns, with aim of obtaining the essential and important flow information. The selection of resolutions depends on the distinct movement frequencies of different flow patterns. We then calculate the weighted network measures for each generated networks. We show the calculated results in Figs. 1-4, where $K_{w}$ represents the fixed water-cut and $V_{m}$ represents mixture flow velocity. When the water-cut and mixture flow velocity are low, e.g., by fixing the $K_{w}=60 \%$ and increasing the $V_{m}$ from $0.0184 \mathrm{~m} / \mathrm{s}$ to $0.2579 \mathrm{~m} / \mathrm{s}$, the oil-water flows gradually evolve from oil-in-water slug flow to oil-in-water bubble flow. For a low mixture flow velocity, the oil-water flows appear as vertical oilin-water slug flow (D OS/W). The nonlinear flow dynamical behavior underlying this flow pattern lies in the nonlinear quasi-periodic movements of large oil slugs, trailed by some small oil droplets. The flow structure of D OS/W flow presents a non-homogeneous distribution characteristic. Specifically, the oil slugs can simultaneously impact more than one channel when the flows pass through the designed sensor. The nonlinear signals from D OS/W flow exhibit the features of chaotic oscillations. Consequently, the network 


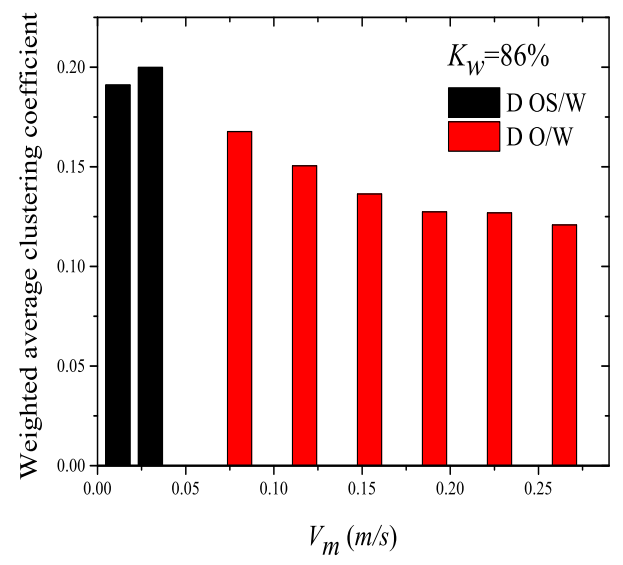

(a)

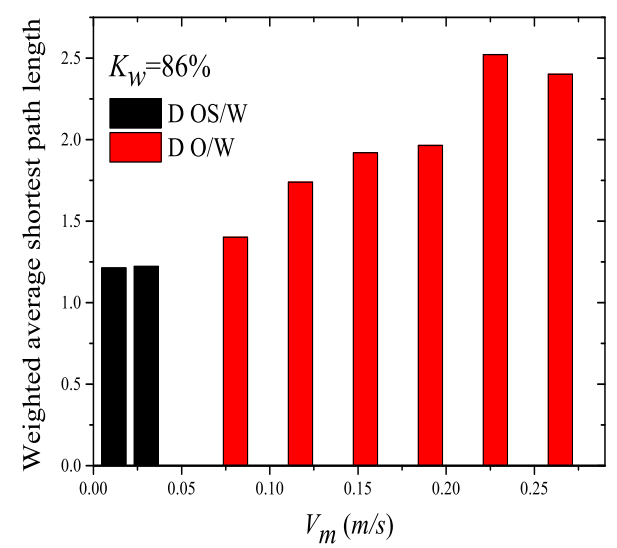

(b)

Fig. 3. Distributions of the network measures with the change of total mixture flow velocity for different flow conditions for a fixed $K_{w}=86 \%$. (a) Weighted average clustering coefficient; (b) Weighted average shortest path length.

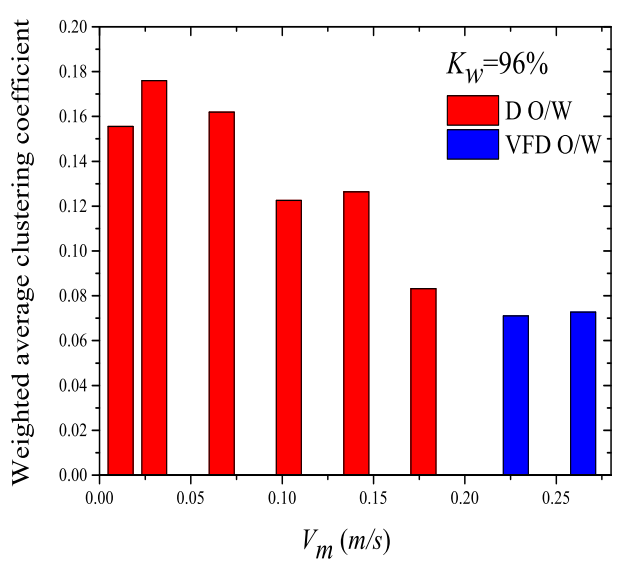

(a)

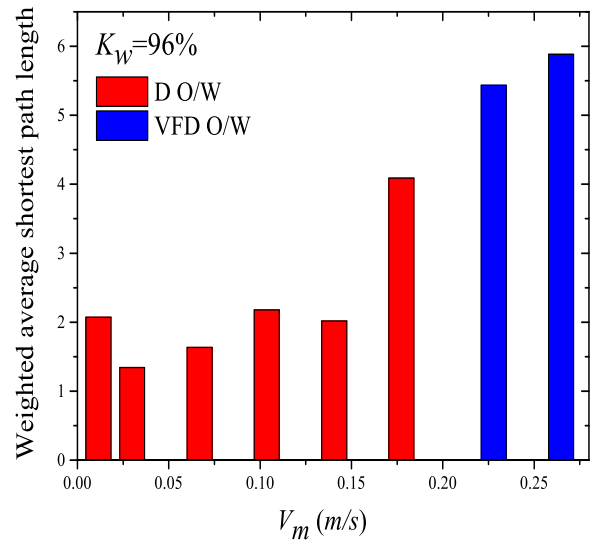

(b)

Fig. 4. Distributions of the network measures with the change of total mixture flow velocity for different flow conditions for a fixed $K_{w}=96 \%$. (a) Weighted average clustering coefficient; (b) Weighted average shortest path length.

from D OS/W flow presents a high value of weighted average clustering coefficient and a low value of weighted average shortest path length. As the mixture flow velocity constantly increases, the turbulence energy is enhanced, leading to the breakage of large oil slugs and the formation of small oil bubbles. That is, a transition from oil-in-water slug flow (D OS/W) to oil-in-water bubble flow (D O/W) occurs. As shown in Figs. 1-3, we find that the measures of WMCN allow efficiently characterizing the underlying nonlinear flow behaviors governing the transition of flow patterns. Specifically, the complexity and nonlinearity of the flow signals gradually become more significant in such a flow transition, induced by the nonlinear dynamical movements of large numbers of oil bubbles. Meanwhile, the non-homogeneous distribution is weakened as the flow evolves from D OS/W flow to D O/W flow. Correspondingly, the connectivity of the network is weakened and the connections between the nodes gradually become sparse. As a result, the weighted average clustering coefficient decreases while weighted average shortest path length increases in the transition from D OS/W flow to D O/W flow. When the water-cut and mixture flow velocity are high, e.g. $K_{w}=96 \%$ and $V_{m}$ exceeds $0.1842 \mathrm{~m} / \mathrm{s}$, the flow pattern evolves from D O/W flow to very fine dispersed bubble flow (VFD $\mathrm{O} / \mathrm{W})$, in which the oil bubbles are further crushed into very fine oil droplets, whose 
diameters are almost equal. The complexity and nonlinearity of the flow signals are further strengthened in this flow transition. As shown in Fig. 4, the weighted average clustering coefficient further decreases and the weighted average shortest path length continually increases in the transition from $\mathrm{D} \mathrm{O} / \mathrm{W}$ flow to VFD $\mathrm{O} / \mathrm{W}$ flow, indicating that a mass of oil droplets flow in a water continuum with a complicated nonlinear dynamics and its flow structure exhibits a feature of homogeneous distribution. These interesting results indicate that the WMCN analysis is capable of characterizing the nonlinear flow behaviors associated with the flow transitions from the multivariate nonlinear time series.

\section{Permutation entropy analysis of nonlinear flow signals}

We calculate the permutation entropy (PE) [Bandt \& Pompe, 2002; Liu et al., 2011] for the multivariate nonlinear flow signals, aiming at interpreting and supporting our MWCN results. The permutation entropy enables to measure the complexity of a univariate time series. The basic idea of the permutation entropy can be described as follows:

Firstly, we embed a one-dimensional time series $\{x(i)\}, i=1,2, \ldots, n$ to a $m$-dimensional space:

$$
X_{i}=[x(i), x(i+\tau), \ldots, x(i+(m-1) \tau)],
$$

where $m$ represents the embedded dimensions and $\tau$ is the delay time. For a given, but otherwise arbitrary $i$, the $m$ number of real value $X_{i}=[x(i), x(i+\tau), \ldots, x(i+(m-1) \tau)]$ can be arranged in an increasing order:

$$
x\left[i+\left(j_{1}-1\right) \tau\right] \leq x\left[i+\left(j_{2}-1\right) \tau\right] \leq \ldots \leq x\left[i+\left(j_{m}-1\right) \tau\right] .
$$

When an equality occurs, e.g., $x\left[i+\left(j_{1}-1\right) \tau\right]=x\left[i+\left(j_{2}-1\right) \tau\right]$, we sort them according to the value of $j_{1}$ and $j_{2}$ by the following rules: If $j_{1}<j_{2}, x\left[i+\left(j_{1}-1\right) \tau\right]<x\left[i+\left(j_{2}-1\right) \tau\right]$; else, $x\left[i+\left(j_{1}-1\right) \tau\right] \geq$ $x\left[i+\left(j_{2}-1\right) \tau\right]$. Thus, for each one-dimensional time series $\{x(i)\}, i=1,2, \ldots, n$, we can obtain a set of symbol sequences:

$$
S(l)=\left(j_{1}, j_{2}, \ldots, j_{m}\right), \quad l=1,2, \ldots, k, k \leq m !
$$

where $S(l)$ represents one of the $m$ ! different symbol sequences of the $m$-dimensional phase space map. The permutation entropy [Bandt \& Pompe, 2002] of time series can be defined as

$$
H_{p}(m)=-\sum_{j=1}^{k} P_{j} \ln P_{j},
$$

where $P_{j}$ represents the probability of occurrence of each symbol sequence. The permutation entropy $H_{p}$ allows measuring the complexity of nonlinear time series.

We calculate the permutation entropy (PE) of 48 channel signals for three different flow patterns and show the results in Fig. 5, where the distribution of permutation entropy is presented in the form of a radar map. As can be seen, the permutation entropies of oil-in-water slug flow (D OS/W) exhibit small values and their distribution is rather non-uniform, indicating the chaotic quasi-periodic dynamics of large oil slugs and heterogeneous distribution of oil phase. For the oil-in-water bubble flow (D O/W), the value of permutation entropy of each channel is slowly increasing, and the difference between the channels is narrowing, suggesting that the heterogeneity of mixture flow is weakened and meanwhile the complexity and nonlinearity of the flow signals become more significant. For the very fine dispersed bubble flow (VFD $\mathrm{O} / \mathrm{W}$ ), the distribution of permutation entropy presents a uniform feature and the values are large, indicating that the flow distribution is homogeneous and the nonlinear dynamics underlying VFD $\mathrm{O} / \mathrm{W}$ flow is most complicated among three vertical oil-water flow patterns. These results well support the findings from WMCN analysis, which renders the WMCN method potentially powerful for realizing multi-channel information fusion. 


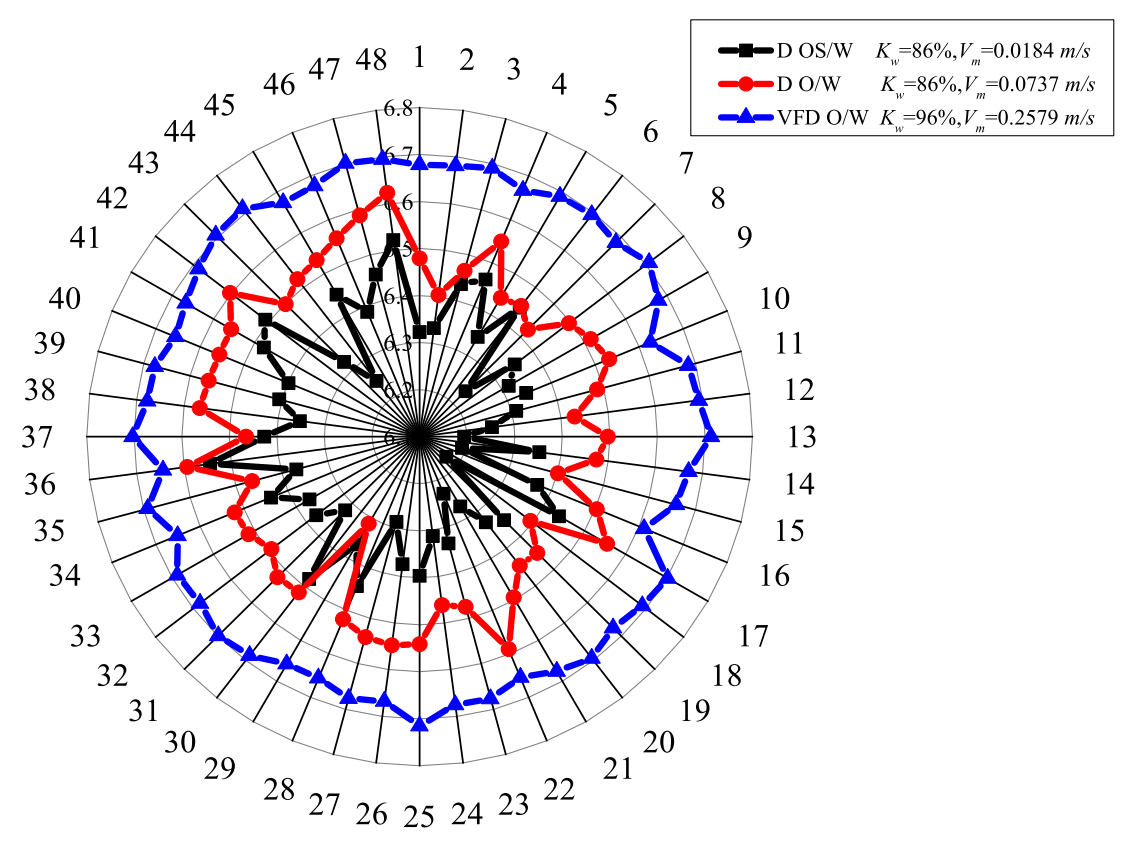

Fig. 5. Distributions of permutation entropy of multivariate nonlinear flow signals for three different flow patterns.

\section{Conclusion}

In summary, we have articulated a novel wavelet multiresolution complex network (WMCN) for analyzing multivariate nonlinear time series. The basic idea of WMCN is to extract wavelet coefficients series at different resolutions from each time series by using wavelet multiresolution decomposition. We then infer the WMCN by regarding each time series as a node and determining the connections in terms of the distance between the feature vectors extracted from wavelet coefficients series. We infer WMCNs from multivariate nonlinear flow signals and use the weighted average clustering coefficient and the weighted average shortest path length to characterize the derived networks. The results suggest that our method is capable of uncovering the chaotic and nonlinear flow behavior governing the transitions of different oilin-water flow patterns at different resolutions. In addition, we calculate the distributions of permutation entropy for multivariate nonlinear flow signals and the results well support the findings from WMCN analysis. Bridging wavelet multiresolution and multivariate complex network provides a novel methodology for analyzing multivariate nonlinear time series which widely exist in nonlinear science and engineering.

\section{Acknowledgments}

Z. K. Gao was supported by National Natural Science Foundation of China under Grant No. 61473203, and the Natural Science Foundation of Tianjin, China under Grant No. 16JCYBJC18200.

\section{References}

Antoniou, I. E. \& Tsompa, E. T. [2008] "Statistical analysis of weighted networks," Discrete Dyn. Nat. Soc. 2008, 375452.

Bandt, C. \& Pompe, B. [2002] "Permutation entropy: a natural complexity measure for time series," Phys. Rev. Lett. 88, 174102.

Boccaletti, B., Bianconi, G., Criado, R., Genio, C. I. D., Gomez-Gardenes, J., Romance, M., SendinaNadal, I., Wang, Z. \& Zanin, M. [2014] "The structure and dynamics of multilayer networks," Phys. Rep. 544, 1-122. 
Daw, C. S., Finney, C. E., Vasudevan, M., Vangoor, N. A., Nguyen, K., Bruns, D. C., Kostelich, E. J., Grebogi, C., Ott, E. \& Yorke, J. A. [1995] "Self-organization and chaos in a fluidized bed," Phys. Rev. Lett 75, 2308-2311.

Deising, D., Marschall, H. \& Bothe, D. [2015] "A unified single-field model framework for VOF-based simulations of interfacial species transfer applied to bubbly flows," Chem. Eng. Sci. 139, 173-195.

Donges, J. F., Donner, R. V., Trauth, M. H., Marwan, N., Schellnhuber, H. J. \& Kurths, J. [2011] "Nonlinear detection of paleoclimate-variability transitions possibly related to human evolution," Proc. Natl. Acad. Sci. USA 108, 20422-20427.

Donges, J. F., Heitzig, J., Donner, R. V. \& Kurths, J. [2012] "Analytical framework for recurrence network analysis of time series," Phys. Rev. E 85, 046105.

Feldhoff, J. H., Donner, R. V., Donges, J. F. Marwan, N. \& Kurths, J. [2012] "Geometric detection of coupling directions by means of inter-system recurrence networks," Phys. Lett. A 376, 3504-3513.

Gao, Z. K., Zhang, X. W., Jin, N. D., Donner, R. V., Marwan, N. \& Kurths, J. [2013] "Recurrence network from multivariate signals for uncovering dynamic behavior of horizontal oil-water stratified flows," EPL 103, 50004.

Gao, Z. K., Fang, P. C., Ding, M. S. \& Jin, N. D. [2015] "Multivariate weighted complex network analysis for characterizing nonlinear dynamic behavior in two-phase flow," Exp. Therm. Fluid Sci. 60, 157-164.

Gao, Z. K., Yang, Y. X., Fang, P. C., Zou, Y. \& Xia, C. Y. [2015] "Multiscale complex network for analyzing experimental multivariate time series," EPL 109, 30005.

Gao, Z. K., Cai, Q., Yang, Y. X., Dang, W. D. \& Zhang, S. S. [2016] "Multiscale limited penetrable horizontal visibility graph for analyzing nonlinear time series," Sci. Rep. 6, 35622.

Gao, Z. K., Yang, Y. X., Zhai, L. S., Jin, N. D. \& Chen, G. R. [2016] "A four-sector conductance method for measuring and characterizing low-velocity oil-water two-phase flows," IEEE Trans. Instrum. Meas. 65, 1690-1697.

Gao, Z. K., Small, M. \& Kurths, J. [2016] "Complex network analysis of time series," EPL 116, 50001.

Gao, Z. K., Cai, Q., Yang, Y. X., Dong, N. \& Zhang, S. S. [2017] "Visibility graph from adaptive optimal kernel time-frequency representation for classification of epileptiform EEG," Int. J. Neural Syst. 27, 1750005.

Goyal, K. \& Mehra, M. [2017] "An adaptive meshfree spectral graph wavelet method for partial differential equations," Applied Numer. Math. 113, 168-185.

Guler, I. \& Ubeyli, E. D. [2005] "Adaptive neuro-fuzzy inference system for classification of EEG signals using wavelet coefficients," J. Neurosci. Meth. 148, 113-121.

He, K. J., Lai, K. K. \& Yen, J. [2012] "Ensemble forecasting of Value at Risk via Multi Resolution Analysis based methodology in metals markets," Expert Syst. Applications 39, 4258-4267.

Huang, L., Lai, Y. C. \& Harrison, M. A. F. [2012] "On some recent advances in complex software networks: modeling, analysis, evolution and applications," Int. J. Bifurcation and Chaos 22, 1250236.

Huang, Z. G., Dong, J. Q., Huang, L. \& Lai, Y. C. [2014] "Universal flux-fluctuation law in small systems," [2014] Sci. Rep. 4, 6787.

Jachan, M., Henschel, K., Nawrath, J., Schad, A., Timmer, J. \& Schelter, B. [2009] "Inferring direct directed-information flow from multivariate nonlinear time series," Phys. Rev. E 80, 011138.

Jammazi, R. [2012] "Oil shock transmission to stock market returns: Wavelet-multivariate Markov switching GARCH approach," Energy 37, 430-454.

Kao, L. J., Chiu, C. C., Lu, C. J. \& Chang, C. H. [2012] "A hybrid approach by integrating wavelet-based feature extraction with MARS and SVR for stock index forecasting," Decision Support Systems 54, 1228-1244.

Kenett, D. Y., Perc, M. \& Boccaletti, S. [2015] "Networks of networks - An introduction," Chaos, Solitons E Fractals 80, 1-6.

Kim, B., Do, Y. \& Lai, Y. C. [2013] "Emergence and scaling of synchronization in moving-agent networks with restrictive interactions," Phys. Rev. E 88, 042818.

Lacasa, L., Luque, B., Ballesteros, F., Luque, J. \& Nuno, J. C. [2008] "From time series to complex networks: The visibility graph," Proc. Natl. Acad. Sci. USA 105, 4972-4975.

Lina, J. M. \& Mayrand, M. [1995] "Complex daubechies wavelets," Appl. Comput. Harmon. A 2, 219-229. 
Liu, C., Zhou, W. X. \& Yuan, W. K. [2010] "Statistical properties of visibility graph of energy dissipation rates in three-dimensional fully developed turbulence," Physica A 389, 2675-2681.

Liu, Y., Chou, T. S., Baek, H., Do, Y., Choi, J. H. \& Chung, Y. D. [2011] "Permutation entroy applied to movement behaviors of drosophila melanogaster," Mod. Phys. Lett. B 25, 1133-1142.

Livi, L., Maiorino, E., Rizzi, A. \& Sadeghian, A. [2016] "On the Long-Term Correlations and Multifractal Properties of Electric Arc Furnace Time Series," Int. J. Bifurcation and Chaos 26, 1650007.

Lu, J. H. \& Chen, G. R. [2005] "A time-varying complex dynamical network models and its controlled synchronization criteria," IEEE Trans. Automat. Contr. 50, 841-846.

Lu, J., Ho, D. W. C., Cao, J. D. \& Kurths, J. [2013] "Single impulsive controller for globally exponential synchronization of dynamical networks," Nonlinear Anal.-Real 14, 581-593.

Majhi, S., Perc, M. \& Ghosh, D. [2016] "Chimera states in uncoupled neurons induced by a multilayer structure," Sci. Rep. 6, 39033.

Marwan, N., Romano, M. C. \& Thiel, M. [2007] "Recurrence plots for the analysis of complex systems," Phy. Rep. 438, 237-329.

Marwan, N., Donges, J. F., Zou, Y., Donner, R. V. \& Kurths, J. [2009] "Complex network approach for recurrence analysis of time series," Phys. Lett. A 373, 4246-4254.

Marwan, N. \& Kurths, J. [2015] "Complex network based techniques to identify extreme events and (sudden) transitions in spatio-temporal systems," Chaos 25, 097609.

Mosdorf, R. \& Górski, G. [2015] "Detection of two-phase flow patterns using the recurrence network analysis of pressure drop fluctuations," Int. Commun. Heat Mass 64, 14-20.

Nakamura, T., Tanizawa, T. \& Small, M. [2016] "Constructing networks from a dynamical system perspective for multivariate nonlinear time series," Phys. Rev. E 93, 032323.

Peng, X. L., Small, M., Xu, X. J. \& Fu, X. [2013] "Temporal prediction of epidemic patterns in community networks," New J. Phys. 15, 113033.

Scarsoglio, S., Iacobello, G. \& Ridolfi, L. [2016] "Complex Networks Unveiling Spatial Patterns in Turbulence," Int. J. Bifurcation and Chaos 26, 1650223.

Schelter, B., Mader, M., Mader, W., Sommerlade, L., Platt, B., Lai, Y. C., Grebogi, C. \& Thiel, M. [2014] "Overarching framework for data-based modelling," EPL 105, 107-110.

Shen, Z. S., Wang, W. X., Fan, Y., Di, Z. R. \& Lai, Y. C. [2014] "Reconstructing propagation networks with natural diversity and identifying hidden sources," Nat. Commun. 5, 4323.

Tang, J. J., Liu, F., Zhang, W. B., Zhang, S. \& Wang, Y. H. [2016] "Exploring dynamic property of traffic flow time series in multi-states based on complex networks: Phase space reconstruction versus visibility graph," Physica A 450, 635-648.

Tee, A. R., Manning, B. D., Roux, P. P., Cantley, L. C. \& Bienis, J. [2011] "Haze detection and removal in high resolution satellite image with wavelet analysis," IEEE Trans. Geosci. Remote 37, 2655-2663.

Wang, H. C., He, K. Q., Li, B. \& Lu, J. H. [2012] "On some recent advances in complex software networks: modeling, analysis, evolution and applications," Int. J. Bifurcation and Chaos 22, 1250024.

Wang, W. X., Yang, R., Lai, Y. C., Kovanis, V. \& Grebogi, C. [2011] "Predicting catastrophes in nonlinear dynamical systems by compressive sensing," Phys. Rev. Lett. 106, 154101.

Wang, W. X., Lai, Y. C. \& Grebogi, C. [2016] "Data based identification and prediction of nonlinear and complex dynamical systems," Phys. Rep. 644, 1-76.

Wang, Z. \& Wang, L. [2015] "Evolutionary games on multilayer networks: A colloquium," Eur. Phys. B 88, 124.

Wang, Z., Bauch, C. T., Bhattacharyya, S., d'Onofrio, A., Manfredi, P., Perc, M., Perra, N., Salathé, M. \& Zhao, D. W. [2016] "Statistical physics of vaccination," Phys. Rep. 664, 1-113.

Xiang, R. X., Zhang, J., Xu, X. K. \& Small, M. [2012] "Multiscale characterization of recurrence-based phase space networks constructed from time series," Chaos 22, 013107.

Xu, X. K., Zhang, J. \& Small, M. [2008] "Superfamily phenomena and motifs of networks induced from time series," Proc. Natl. Acad. Sci. USA 105, 19601-19605.

Zhang, J. \& Small, M. [2006] "Complex network from pseudoperiodic time series: topology versus dynamics," Phys. Rev. Lett. 96, 238701.

Zhang, J., Sun, J. F., Luo, X. D., Zhang, K., Nakamura, T. \& Small, M. [2008] "Characterizing pseudope- 
riodic time series through the complex network approach," Physica D 237, 2856-2865.

Zhang, J., Cheng, W., Liu, Z. W., Zhang, K., Lei, X., Yao, Y., Becker, B., Liu, Y. C., Kendrick, K. M., Lu, G. M. \& Feng, J. F. [2016] "Neural electrophysiological and anatomical basis of brian-network variability and its characteristic changes in mental disorders," Brain 139, 1-15.

Zou, W., Senthilkumar, D. V., Nagao, R., Kiss, I. Z., Tang, Y., Koseska, A., Duan, J. Q. \& Kurths, J. [2015] "Restoration of rhythmicity in diffusively coupled dynamical networks," Nat. Commun. 6, 8709.

Zou, Y., Small, M., Liu, Z. \& Kurths, J. [2014] "Complex network approach to characterize the statistical features of the sunspot series," New J. Phys. 16, 013051. 